\title{
COVID-19: Magnifying the Effect of Health Disparities
}

\author{
Eun Ji Kim ${ }^{1,2}$ (1) , Lyndonna Marrast ${ }^{1,2}$, and Joseph Conigliaro ${ }^{1,2}$
}

'Division of General Internal Medicine, Donald and Barbara Zucker School of Medicine at Hofstra/Northwell 2001 Marcus Avenue Suite S160, Lake Success, NY, USA; ${ }^{2}$ Feinstein Institutes of Medical Research Manhasset, NY, USA.

$J$ Gen Intern Med 35(8):2441-2

DOI: $10.1007 / \mathrm{s} 11606-020-05881-4$

(c) Society of General Internal Medicine 2020

$\mathrm{C}$ oronavirus disease 19 (COVID-19) is the condition caused by severe acute respiratory syndrome coronavirus 2 (SARS-2-CoV-2) infections which is now prevalent in all 50 states with clustering of cases in major cities in New York, Michigan, California, and New Jersey. COVID-19 that was initially thought to be the great equalizer-no discrimination with who can become infected - now reveals patterns of disparities for low-income persons and racial and ethnic minority populations. This novel coronavirus which results in increased rates of respiratory failure and death among infected individuals $^{1,2}$ is found to disproportionally affect those with comorbidities such as diabetes, heart disease, and asthma. These conditions are known to disproportionately affect minorities who are already impacted by health disparities.

Even before COVID-19, health disparities were pervasive in the USA. We define disparities as differences in healthcare utilization and health outcomes among distinct social groups. ${ }^{3}$ Emerging data show that racial/ethnic minorities and those from the working class have worse health outcomes due to COVID-19. In Chicago, Blacks comprise $30 \%$ of the city's population but $70 \%$ of COVID-19 deaths. Based on our personal experience and data from our health system, COVID-19 is uncovering disparities embedded within our society. In this paper, we identify areas in which COVID-19 is exacerbating existing disparities and offer potential solutions to address these disparities.

Structural barriers contributed to disparities in COVID-19 testing and diagnosis in the early weeks of the pandemic. One of the major issues with diagnosing COVID-19 is limited testing availability. Initially, testing was centralized and there were few testing sites. To control the number of patients presenting for tests, residents in New York were required to

The views expressed in this article are those of the authors and do not necessarily represent the views of the Donald and Barbara Zucker School of Medicine at Hofstra/Northwell, Feinstein Institutes of Medical Research.

Received April 14, 2020

Revised April 14, 2020

Accepted April 28, 2020

Published online May 11, 2020 obtain a doctor's prescription. Previous studies have shown that racial/ethnic minorities are less likely to have access to a primary care provider. ${ }^{4}$ Thus, this requirement may have served as structural barrier in obtaining COVID-19 testing. Then, the state implemented "drive-thru" testing to increase the number of patients receiving tests but this required access to a vehicle. Though the intention was to control the number of patients getting tested through prescriptions and "drive-thru" testing, patients without a doctor or without access to a vehicle were less likely to be tested. Controlling access to tests in this way likely disadvantaged racial and ethnic minorities and those who rely on public transportation. Furthermore, if a patient has a high clinical suspicion for COVID-19 infection but lacks a primary care provider or vehicle, their only recourse is to seek testing in an emergency room. Given the cost of emergency room copays, some patients have to decide whether to pay to get evaluated for testing or monitor their symptoms at home. Additionally, COVID-19 results are available 7 to 10 days after and during this time, patients again encounter a dilemma whether to quarantine themselves at home or go to work. Therefore, having an established policy for free and easily accessible COVID-19 testing would reduce the impact of the structural barriers to testing and, in turn, minimize disparities.

We are witnessing unintended consequences of the COVID-19 outbreak; patients with non-COVID-19 medical conditions avoid healthcare to reduce the risk of COVID-19 exposure. As hospitals are filled with patients who test positive for COVID-19, COVID-19-negative patients with existing medical conditions are more susceptible to complications of those conditions. Cardiologists are noticing a reduction in the number of patients with heart attacks and those who are presenting with heart attacks are having worse outcomes. Surgeons also observe patients that require surgical interventions are presenting with gangrene or with increased risk of surgical complications. Patients from racial and ethnic minorities have an increased prevalence of common medical conditions compared to non-Hispanic Whites, and a high percentage of them have undiagnosed common medical conditions. ${ }^{4}$ To reduce disparities arising from the management of both COVID-19 and non-COVID disease outcomes, it is critical to develop testing to rapidly assess COVID-19 status, isolate COVID-19 patients from non-COVID patients, and efficiently manage non-COVID-19 diseases. 
Public health recommendations to contain COVID-19 can worsen disparities due to a lack of resources and underlying institutional racism. To reduce the spread of COVID-19, states have implemented various procedures, including social distancing, wearing masks in public areas, working from home, and closing schools. These recommendations seem sensible for containing the disease but may be difficult to implement depending on one's social situation. Access to masks has been difficult given the nationwide shortage of masks. Also, wearing a mask has been associated with having the diseases and, in some instances, society may perceive criminality (when none exists). When the government communicates ambiguous messages about wearing masks to reduce COVID-19 infection, it is difficult for the public, especially racial and ethnic minorities and the working class, to interpret and adopt the recommendation. Therefore, it is important to have clear recommendations to ensure general public safety.

Social distancing has contributed to decreased expected mortality, a positive outcome, but negative impacts will be the hardest among the working class. In New York, bus drivers limit the number of passengers riding each bus to respect social distancing rules. During a phone conversation, a patient stated that this practice would effectively double his travel time to the clinic for a doctor's visit. He usually rides two buses, but with limits to the maximum number of people allowed, he more often needs to wait for the next bus. Considering that New York City public transportation is now running on a weekend schedule, those who rely on public transportation experience significantly longer travel times and an increased risk of exposure during the pandemic. This becomes more problematic if someone is an asymptomatic carrier of the virus.

Lastly, we are anticipating significant disparities arising from the lack of health insurance due to one of the highest unemployment rates in history. In the coming months, this will have a significant impact on healthcare utilization and health outcomes. The longer the COVID-19 outbreak persists, the more employees will be at a risk of becoming furloughed. In the beginning of the outbreak, a television news anchor suggested that people should receive their health checkups now while they still have their health insurance as the majority of people in the USA obtain their health insurance through their employer. Given the increase in unemployment, it is unclear what will happen in situations when these uninsured patients need medical care. Also, those without health insurance will no longer receive routine checkups, cancer screening, and disease management visits. This likely will result in a widening of the disparity gap in the long term, especially among vulnerable populations.

Though present before the pandemic, health disparities may increase after our society begins to recover from COVID-19. Until COVID-19 becomes eradicated or a vaccine is developed, society must combat this disease as one to minimize mortality, complications, and social consequences (i.e., unemployment) arising from this disease. It is imperative that rapid COVID-19 screening be freely available to the general public and those who have an increased risk of exposure. Only then can we elevate the care for all medical conditions and mitigate the health disparities arising from COVID-19. Though COVID-19 is not the great equalizer based on disparities in outcomes, our response can result in more equitable access to care for all segments of the population.

Corresponding Author: Eun Ji Kim, Division of General Internal Medicine, Donald and Barbara Zucker School of Medicine at Hofstra/ Northwell 2001 Marcus Avenue Suite S160, Lake Success, NY 11042, USA (e-mail: ekim7@northwell.edu).

Funding Information Dr. Lyndonna Marrast is funded by NLM grant (3R01LM012836-03S1).

\section{Compliance with Ethical Standards:}

Conflict of Interest: The authors declare that they do not have a conflict of interest.

\section{REFERENCES}

1. Wu C, Chen X, Cai Y, et al. Risk Factors Associated With Acute Respiratory Distress Syndrome and Death in Patients With Coronavirus Disease 2019 Pneumonia in Wuhan, China. JAMA Internal Medicine. 2020.

2. Zhou F, Yu T, Du R, et al. Clinical course and risk factors for mortality of adult inpatients with COVID-19 in Wuhan, China: a retrospective cohort study. The Lancet.

3. Kilbourne AM, Switzer G, Hyman K, Crowley-Matoka M, Fine MJ. Advancing health disparities research within the health care system: a conceptual framework. Am J Public Health. 2006;96(12):2113-2121.

4. Kim EJ, Kim T, Conigliaro J, Liebschutz JM, Paasche-Orlow MK, Hanchate AD. Racial and Ethnic Disparities in Diagnosis of Chronic Medical Conditions in the USA. J Gen Intern Med. 2018;33(7):1116-1123.

Publisher's Note: Springer Nature remains neutral with regard to jurisdictional claims in published maps and institutional affiliations. 